\title{
BMJ
}

\section{Introduction of laparoscopic bariatric surgery in England: observational population cohort study}

\begin{abstract}
Elaine M Burns, clinical research fellow, ${ }^{1}$ Haris Naseem, core surgical trainee, ${ }^{1}$ Alex Bottle, lecturer in medica statistics, ${ }^{2}$ Antonio Ivan Lazzarino, honorary clinical lecturer, ${ }^{2}$ Paul Aylin, clinical reader in epidemiology and public heath, ${ }^{2}$ Ara Darzi, professor of surgery, ${ }^{1}$ Krishna Moorthy, senior lecturer and honorary consultant gastrointestinal surgeon, ${ }^{1}$ Omar Faiz, senior lecturer and honorary consultant gastrointestinal surgeon ${ }^{1}$
\end{abstract}

\begin{abstract}
'Department of Surgery, Imperial College, St Mary's Hospital, London W21NY

${ }^{2}$ Foster Unit, Department of Primary Care and Social Medicine, Imperial College, London

EC1A 9LA

Correspondence to: 0 Faiz, Department of Biosurgery and Surgical Technology, Imperial College, St Mary's Hospital, London W21NY omarfaiz@aol.com
\end{abstract}

Cite this as: BMJ 2010;341:C4296 doi:10.1136/bmj.c4296

\section{ABSTRACT}

Objectives To describe national trends in bariatric surgery and examine the factors influencing outcome in bariatric surgery in England.

Design Observational population cohort study. Setting Hospital Episode Statistics database.

Participants All patients who had primary gastric bypass, gastric banding, or sleeve gastrectomy procedures between April 2000 and March 2008.

Main outcome measures 30 day mortality, mortality at one year after surgery, unplanned readmission to hospitalwithin 28 days, and duration of stay in hospital. Results 6953 primary bariatric procedures were carried out during the study period, of which 3649 were gastric band procedures, 3191 were gastric bypass procedures, and 113 were sleeve gastrectomy procedures. A marked increase occurred in the numbers of bariatric procedures done, from 238 in 2000 to 2543 in 2007, with an increase in the percentage of laparoscopic procedures over the study period (28\% (66/238) laparoscopic procedures in 2000 compared with $74.5 \%$ (1894/2543) in 2007). Overall, $0.3 \%$ (19/6953) patients died within 30 days of surgery. The median length of stay in hospital was 3 (interquartile range 2-6) days. An unplanned readmission to hospital within 28 days of surgery occurred in $8 \%$ (556/ 6953) of procedures. No significant increase in mortality or unplanned readmission was seen over the study period, despite the exponential increase in minimal access surgery and consequently bariatric surgery. Conclusions Bariatric surgery has increased exponentially in England. Although postoperative weight loss and reoperation rates were not evaluated in this observational population cohort study, patients selected for gastric banding had lower postoperative mortality and readmission rates and a shorter length of stay than did those selected for gastric bypass.

\section{INTRODUCTION}

Obesity is a global concern. The World Health Organization predicts that by 2015,700 million of the world's population will be obese, as defined by a body mass index of at least 30 (calculated as weight $(\mathrm{kg}) /$ height $\left.(\mathrm{m})^{2}\right){ }^{1}$
Based on surgical techniques originating in the 1950s, bariatric surgery has developed considerably and has increased in popularity in recent years. ${ }^{2}$ Procedures have traditionally been described as being either restrictive or malabsortive,${ }^{3}$ but a greater understanding of the pathophysiology of obesity, including the role of gut hormones, has showed this to be a rather simplistic view of the various procedures. ${ }^{4}$ Use of minimally invasive techniques has increased significantly in recent years and is now recognised as standard practice in most Western countries. ${ }^{25}$

Health consequences attributed to obesity are well documented and involve physical, psychological, and social consequences. ${ }^{16}$ After bariatric surgery, patients have been shown to have an improvement or a complete resolution of their comorbidities. ${ }^{7}$ Bariatric surgery has been shown to reduce the risk of death, hospital admission, and long term cost to the health service ${ }^{8-10}$ In the United Kingdom, the National Institute for Health and Clinical Excellence (NICE) published guidance on the use of bariatric surgery in 2002. ${ }^{11}$ Morbid obesity was defined as a body mass index exceeding 40 or a body mass index of 35 or greater with significant coexisting disease that could be improved by weight loss. A systematic review has concluded that surgery is superior to conventional treatment in reducing weight. However, the review failed to show the superiority of one surgical method over the others. ${ }^{12}$

Ells and colleagues investigated the demographics of patients who had bariatric surgery in England between 1996 and 2005. ${ }^{13}$ They identified significant variation in the regional provision of this service. Little is known about the trends and demographics of bariatric surgery in England in more recent years. The aim of this study was to investigate national trends in the contemporary use of bariatric surgery in England. In particular, we sought to describe the use of different surgical techniques, as well as the uptake of laparoscopic surgery, and to examine the factors influencing postoperative outcome after bariatric surgery. 


\section{METHODS}

\section{Hospital Episode Statistics}

The Hospital Episode Statistics (HES) dataset has been described previously. ${ }^{14}$ In brief, this dataset is nationally collected and incorporates all admissions to NHS trusts in England (a trust may comprise more than one hospital site). Patients treated in non-NHS hospitals are not included in this dataset. During the period of this study, no national database included all bariatric patients in the independent sector and the NHS in England. HES data include diagnostic, procedural, and demographic information. The finished consultant episode represents the time a patient spends under the care of a specialist or other allied health professional. These episodes may be linked into a single admission. A patient is assigned a primary diagnosis code and up to 13 secondary diagnoses codes by using the ICD-10 (international classification of diseases, 10th revision). Up to 12 procedural codes are assigned by using the Office of Population Censuses and Surveys Classification of Surgical Operations and Procedures, 4th edition (OPCS-4.4, introduced in 2007). Earlier editions of OPCS were used for admissions before the OPCS4.4 was introduced. Data on patients' death after admission is available through a link between the HES database and the Office of National Statistics. ${ }^{15}$

The Carstairs index is used to measure socioeconomic deprivation. This is based on a patient's postcode and specifically uses four variables: unemployment, overcrowding, car ownership, and social class. Comorbidity is measured with the Charlson comorbidity index, which was originally validated against mortality. ${ }^{16}$

\section{Inclusions}

We included in the study all adult patients who had a primary elective bariatric procedure (gastric bypass, gastric banding, or sleeve gastrectomy) with a primary diagnosis of obesity between April 2000 and March 2008. We used the following ICD-10 diagnosis codes to denote obesity: E66.0-obesity due to excess calories, E66.1-drug induced obesity, E66.2-extreme obesity and hypoventilation, E66.8 - other obesity, and E66.9-obesity unspecified.

We used OPCS-4 codes to select those patients who had a primary bariatric procedure with a primary diagnosis of obesity. We used separate codes to define three types of bariatric surgery: gastric bypass, sleeve gastrectomy, and gastric banding (table 1). A separate code for sleeve gastrectomy was introduced in OPCS

Table 1|ICD-10 (international classification of diseases, 10th revision) codes used to define three types of bariatric surgery

\begin{tabular}{ll} 
Primary bariatric procedure & ICD-10 codes \\
Gastric bypass & $\mathrm{G} 281, \mathrm{G} 282, \mathrm{G} 283, \mathrm{G} 288, \mathrm{G} 289, \mathrm{G} 311, \mathrm{G} 312, \mathrm{G} 313, \mathrm{G} 314, \mathrm{G} 315$, \\
& $\mathrm{G} 316, \mathrm{G} 318, \mathrm{G} 319, \mathrm{G} 310, \mathrm{G} 320, \mathrm{G} 321, \mathrm{G} 322, \mathrm{G} 323, \mathrm{G} 324, \mathrm{G} 325$, \\
& $\mathrm{G} 328, \mathrm{G} 329, \mathrm{G} 330, \mathrm{G} 331, \mathrm{G} 332, \mathrm{G} 333, \mathrm{G} 335, \mathrm{G} 336, \mathrm{G} 338, \mathrm{G} 339$ \\
\hline Sleeve gastrectomy* & $\mathrm{G} 285$ \\
\hline Gastric banding & $\mathrm{G} 301, \mathrm{G} 302, \mathrm{G} 303, \mathrm{G} 304, \mathrm{G} 308, \mathrm{G} 309$ \\
\hline
\end{tabular}

*G285 was introduced in April 2006.
4.3 (April 2006). We used laparoscopic codes (Y508other specified approach through abdominal cavity, Y75-minimal access approach to abdominal cavity) to differentiate laparoscopic from open procedures. We did not include other bariatric procedures such as biliopancreatic diversion because of disparate coding for these procedures.

We divided patients into three age groups: 17-40 years, 41-60 years, and older than 60 years. The Carstairs deprivation index allowed us to classify patients into five groups (1-5, in order of increasing socioeconomic deprivation; category 6 denotes patients with no Carstairs index recorded). We divided patients into two groups on the basis of their Charlson comorbidity index: those with a score of $0-2$ and those scoring greater than 2 .

\section{Outcome measures}

We defined readmission as an unplanned admission within 28 days of discharge after the primary bariatric procedure. We studied predictors of in-hospital mortality at 30 days and total mortality at 365 days. Data on deaths at 365 days were available from April 2000 to March 2005, so we did not include patients admitted after March 2005 in the analysis of 365 day mortality. Length of stay refers to the number of nights spent in hospital on the primary admission.

\section{Provider volume}

We defined providers as NHS trusts doing primary bariatric procedures from 2000 to 2007 . We ranked patients into equal thirds of provider volume, giving low volume, medium volume, and high volume groups, according to the total caseload during the study period. We therefore considered provider volume as a categorical variable. This approach has been used previously in other outcome studies that have considered volume in the absence of a priori data. ${ }^{17-20}$

\section{Statistical methods}

We used SPSS 17.0 for statistical analyses. We considered $\mathrm{P}$ values $<0.05$ to be significant. We used $\chi^{2}$ analyses to investigate categorical variables. We investigated continuous variables that were normally distributed by using the $t$ test and analysis of variance. We analysed non-parametric data by using MannWhitney, Kruskal-Wallis, and Spearman's tests. We used multiple logistic regression analyses to investigate independent predictors of readmission within 28 days and 30 day mortality. We included in the multiple regression analysis those variables that showed a level of significance of $\mathrm{P}<0.1$. We logarithmically transformed data on length of stay before the regression analysis.

\section{RESULTS}

Between April 2000 and March 2008, 6953 adults had a primary elective bariatric procedure. Of these, 3191 patients had gastric bypass, 3649 had a gastric banding 
Table 2 | Patients' characteristics by type of operation. Values are numbers (percentages) unless stated otherwise

\begin{tabular}{|c|c|c|c|c|c|}
\hline Characteristic & Gastric bypass & Gastric banding & Sleeve gastrectomy & Total & $P$ value* \\
\hline Total number & 3191 (45.9) & $3649(52.5)$ & $113(2)$ & $6953(100)$ & \\
\hline Mean (SD) age (years) & $42.25(9.50)$ & $42.44(9.79)$ & $44.18(8.96)$ & $42.38(9.65)$ & 0.364 \\
\hline Female sex & $2571(80.6)$ & $2968(81.3)$ & $76(67)$ & $5615(80.8)$ & 0.001 \\
\hline \multicolumn{6}{|l|}{ Comorbidity score: } \\
\hline Charlson score $\leq 2$ & $3179(99.6)$ & 3637 (99.7) & $111(98)$ & $6927(99.6)$ & \multirow{2}{*}{0.047} \\
\hline Charlson score $>2$ & $12(0.4)$ & $12(0.3)$ & $2(2)$ & $26(0.4)$ & \\
\hline \multicolumn{6}{|c|}{ Carstairs index of social deprivation: } \\
\hline 1 (least deprived) & $352(11.0)$ & $459(12.6)$ & $6(5)$ & $817(11.8)$ & \multirow{6}{*}{0.011} \\
\hline 2 & $480(15.0)$ & $607(16.6)$ & $13(12)$ & $1100(15.8)$ & \\
\hline 3 & $580(18.2)$ & $675(18.5)$ & $23(20)$ & $1278(18.4)$ & \\
\hline 4 & $797(25.0)$ & $878(24.1)$ & $28(25)$ & 1703 (24.5) & \\
\hline 5 (most deprived) & 975 (30.6) & $1026(28.1)$ & $42(37)$ & $2043(29.4)$ & \\
\hline Unclassified & $7(0.2)$ & $12(0.3)$ & $1(1)$ & $12(0.2)$ & \\
\hline Laparoscopy rate & $1579(49.5)$ & 2768 (75.9) & 89 (79) & $4436(63.8)$ & $<0.001$ \\
\hline
\end{tabular}

procedure, and 113 patients had a sleeve gastrectomy. The code for sleeve gastrectomy was introduced in April 2006.

During the study period, 85 providers carried out between 1 and 604 procedures each; 154 consultant surgeons did between 1 and 552 procedures each over the study period. We divided NHS hospitals into thirds according to their total caseload over the eight year study period. After ranking patients according to the total volume of procedures carried out by their provider, we considered a low volume provider to be a hospital trust that did 188 procedures or fewer and a high volume provider to be a trust that did more than 384 procedures over the study period. We considered the remaining trusts to be medium volume providers. Table 2 shows the characteristics of the patients who had bariatric surgery.

\section{Trends over time}

Patients' mean age at surgery increased slightly over time from 40 (SD 9.39) years to 43 (9.97) years $(\mathrm{P}<0.001)$. We found a marked increase in the number of bariatric procedures carried out during the study period from 238 in $2000-1$ to 2543 to $2007-8$ (figure). The figure shows changing trends in the use of open and laparoscopic procedures, with a substantial increase in the use of laparoscopy over time. In 2000, $28 \%(66 / 238)$ of bariatric procedures were done laparoscopically. By 2007, 74.5\% (1894/2543) of procedures were laparoscopic.

\section{Mortality outcomes}

Overall, 30 day postoperative mortality was $0.3 \%$ (19/ 6953). Between April 2000 and March 2005 (that is, when 365 day postoperative mortality data were available), one year mortality was $1.3 \%(24 / 1866)$. Table 3 shows the 30 day and 365 day mortality, readmission rate, and length of stay by operation. As the sleeve gastrectomy code was introduced in 2006, the 365 day mortality is not available for these patients.
The risk of postoperative mortality was higher among patients with comorbidity (Charlson score $>2$, $8 \%(2 / 26) \quad v$ Charlson score $\leq 2,0.2 \%$ (17/6927); $\mathrm{P}=0.002)$. Patients from areas of higher social deprivation had higher 365 day mortality (Carstairs 5 (most deprived) 3\% (15/495) v Carstairs 1 (least deprived) $1 \%(3 / 239) ; \mathrm{P}=0.004)$. This association was not evident for 30 day mortality $(\mathrm{P}=0.219)$.

Thirty day mortality was lower after laparoscopic surgery compared with open surgery (laparoscopic surgery $0.2 \% \quad(7 / 4436) \quad v$ open surgery $0.5 \% \quad(12 / 2517)$; $\mathrm{P}=0.014)$. Table 4 shows the characteristics and outcomes of patients who had laparoscopic and open bariatric procedures. Sex and age were not associated with either 30 day mortality (sex $\mathrm{P}=0.841$; age $\mathrm{P}=0.900$ ) or 365 day mortality (sex $\mathrm{P}=0.122$; age $\mathrm{P}=0.693$ ).

We found no variation over the study period in postoperative mortality $(\mathrm{P}=0.618)$, mortality at one year $(\mathrm{P}=0.614)$, or readmissions $(\mathrm{P}=0.817)$.Operation type and comorbidity score were predictors of postoperative mortality in multiple logistic regression analysis (table 5).

\section{8 day unplanned readmission and length of stay}

The overall median length of stay was 3 (interquartile range 2-6) days, and the readmission rate was $8 \%$

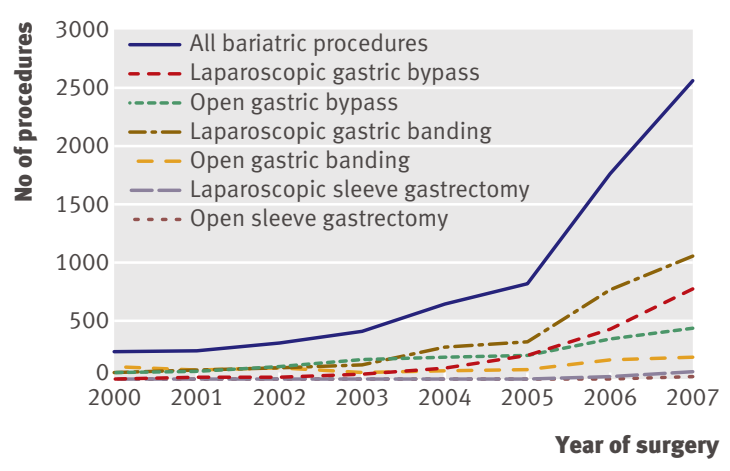

Changes in type of operation over time and trends in uptake of laparoscopic surgery 
Table $3 \mid$ Outcome by type of operation. Values are numbers (percentages) unless stated otherwise

\begin{tabular}{|c|c|c|c|c|c|}
\hline Outcome & $\begin{array}{c}\text { Gastric bypass } \\
(n=3191)\end{array}$ & $\begin{array}{l}\text { Gastric banding } \\
\quad(\mathrm{n}=3649)\end{array}$ & $\begin{array}{l}\text { Sleeve gastrectomy } \\
\qquad(n=113)\end{array}$ & $\begin{array}{c}\text { Total } \\
(\mathrm{n}=6953)\end{array}$ & Pvalue* \\
\hline 30 day mortality & $15(0.5)$ & $3(0.1)$ & $1(1)$ & $19(0.3)$ & 0.004 \\
\hline 365 day mortality & $13 / 786(1.7)$ & $11 / 1080(1.0)$ & NA & 24/1866 (1.3) & 0.229 \\
\hline 28 day readmission rate & $308(9.7)$ & $232(6.4)$ & $16(14)$ & $556(8.0)$ & $<0.001$ \\
\hline Median (interquartile range) length of stay (days) & $5(3-7)$ & $2(1-3)$ & $4(3-7)$ & $3(2-6)$ & $<0.001$ \\
\hline
\end{tabular}

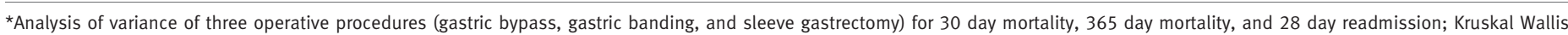
of three operative procedures for length of stay.

(556/6953). Women were less likely than men to be readmitted within 28 days (women $7.7 \%(430 / 5615) v$ men 9.4\% (126/1338); $\mathrm{P}=0.033)$. Patients who had sleeve gastrectomy were readmitted more often than were other patients $(\mathrm{P}<0.001)$, as shown in table 3 . Medium volume trusts tended to readmit patients less often than did other trusts (low volume 8.6\% (212/2479) $v$ medium volume $6.9 \%(164 / 2365)$ v high volume $8.5 \%$ (180/2109); $\mathrm{P}=0.064)$. Patients who had laparoscopic surgery were less likely to be readmitted than were those who had open procedures (laparoscopic 6.6\% $(291 / 4436) v$ open $10.5 \%(265 / 2517) ; \mathrm{P}<0.001)$. The 28 day readmission rate did not increase over time $(\mathrm{P}=0.817)$. Type of operation and open technique were independent predictors of readmission (table 5).

The extent of comorbidity was a significant predictor of hospital stay (Charlson score $\leq 2,3$ (interquartile range 2-6) days $(\mathrm{n}=6927)$ v Charlson score $>2,5(3-8)$ days $(\mathrm{n}=26) ; \mathrm{P}=0.039)$. Women had a shorter length of stay than did men (women $3(2-6)$ days $(n=5615) v$ men
4 (1-6) days $(\mathrm{n}=1338) ; \mathrm{P}=0.017)$. Length of in-hospital stay decreased significantly over the study period (relative risk $0.89,95 \%$ confidence interval 0.88 to 0.89 ; $\mathrm{P}<0.001)$. Patients who had laparoscopic surgery had a significantly shorter length of stay than did those who had an open procedure (laparoscopic 2 (4-8) days $(\mathrm{n}=4436) v$ open $6(1-4)$ days $(\mathrm{n}=2517) ; \mathrm{P}<0.001)$. Patients treated in medium volume trusts had the shortest length of stay (low volume $4(2-6)$ days $(\mathrm{n}=2365)$ v medium volume $3(1-5)$ days $(\mathrm{n}=2365)$ v high volume 4 (2-6) days $(\mathrm{n}=2109) ; \mathrm{P}<0.001)$.

On multiple linear regression analysis, sex, type of operation, laparoscopic approach, age, comorbid status, social deprivation, and trust and consultant volume were predictors of length of stay (table 6).

\section{Gastric bypass versus gastric banding}

Gastric banding and gastric bypass are the two most prevalent bariatric procedures in England; gastric bypass procedures increased as a proportion of the

Table 4| Patients' characteristics and outcomes after gastric bypass and gastric banding surgery, and for all patients who had laparoscopic and open surgery. Values are numbers (percentages) unless stated otherwise

\begin{tabular}{|c|c|c|c|c|c|c|}
\hline & $\begin{array}{l}\text { Gastric bypass } \\
\quad(n=3191)\end{array}$ & $\begin{array}{l}\text { Gastric banding } \\
\quad(n=3649)\end{array}$ & $P$ value & $\begin{array}{l}\text { Open approach } \\
\quad(n=2517)\end{array}$ & $\begin{array}{l}\text { Laparoscopic approach } \\
\qquad(n=4436)\end{array}$ & $P$ value \\
\hline Mean (SD) $(95 \% \mathrm{Cl})$ age (years) & $\begin{array}{c}42.25(9.50) \\
(41.92 \text { to } 42.58)\end{array}$ & $\begin{array}{c}42.44(9.79) \\
(42.13 \text { to } 42.76)\end{array}$ & 0.413 & $\begin{array}{c}42.23(9.51) \\
(41.86 \text { to } 42.60)\end{array}$ & $\begin{array}{c}42.47(9.72) \\
(42.19 \text { to } 42.76)\end{array}$ & 0.308 \\
\hline Female sex & $2571(80.6)$ & $2968(81.3)$ & 0.420 & $2023(80.4)$ & $3592(81.0)$ & 0.542 \\
\hline \multicolumn{7}{|l|}{ Comorbidity score: } \\
\hline Charlson score $\leq 2$ & $3179(99.6)$ & 3637 (99.7) & \multirow{2}{*}{0.742} & $2502(99.4)$ & $4422(99.7)$ & \multirow{2}{*}{0.290} \\
\hline Charlson score $>2$ & $12(0.4)$ & $12(0.3)$ & & $12(0.5)$ & $14(0.3)$ & \\
\hline \multicolumn{7}{|l|}{ Carstairs index of social deprivation: } \\
\hline 1 (least deprived) & $352(11.0)$ & $459(12.6)$ & \multirow{6}{*}{0.041} & $276(11.0)$ & $541(12.2)$ & \multirow{6}{*}{0.056} \\
\hline 2 & $480(15.0)$ & $607(16.6)$ & & $374(14.9)$ & $726(16.4)$ & \\
\hline 3 & $580(18.2)$ & $675(18.5)$ & & $457(18.2)$ & $821(18.5)$ & \\
\hline 4 & $797(25.0)$ & $878(24.1)$ & & $654(26.0)$ & $1049(23.6)$ & \\
\hline 5 (most deprived) & $975(30.6)$ & $1026(28.1)$ & & $749(29.8)$ & $1294(29.2)$ & \\
\hline Unclassified & $7(0.2)$ & $12(0.3)$ & & $7(0.3)$ & $5(0.1)$ & \\
\hline Laparoscopy rate & $1579(49.5)$ & $2768(75.9)$ & 0.001 & NA & NA & NA \\
\hline \multicolumn{7}{|l|}{ Provider volume: } \\
\hline Low (1-188 procedures) & $971 / 2423(40.1)$ & $1452 / 2423(59.9)$ & \multirow{3}{*}{$<0.001$} & $884 / 2479(35.7)$ & $1595 / 2479(64.3)$ & \multirow{3}{*}{$<0.001$} \\
\hline Medium (189-384 procedures) & $782 / 2338(33.4)$ & $1556 / 2338(66.6)$ & & $699 / 2365(29.6)$ & $1666 / 2365(70.4)$ & \\
\hline High (>384 procedures) & $1438 / 2079(69.2)$ & $641 / 2079(30.8)$ & & $934 / 2109(44.3)$ & $1175 / 2109(55.7)$ & \\
\hline 30 day mortality & $15 / 3191(0.5)$ & $3 / 3649(0.1)$ & 0.002 & $12 / 2517(0.5)$ & $7 / 4436(0.2)$ & 0.014 \\
\hline 365 day mortality* & $13 / 786(1.7)$ & $11 / 1080(1.0)$ & 0.229 & $17 / 1047(1.6)$ & $7 / 812(0.9)$ & 0.143 \\
\hline 28 day readmission rate & $308 / 3191(9.7)$ & $232 / 3649(6.4)$ & $<0.001$ & $265 / 2517(10.5)$ & $291 / 4436(6.6)$ & $<0.001$ \\
\hline Median (interquartile range) length of stay (days) & $5(3-7)$ & $2(1-3)$ & $<0.001$ & $6(4-8)$ & $2(1-4)$ & $<0.001$ \\
\hline
\end{tabular}


Table 5 Multiple logistic regression analysis of $\mathbf{3 0}$ day mortality and $\mathbf{2 8}$ day readmission, including all patients

\begin{tabular}{|c|c|c|c|c|}
\hline \multirow[b]{2}{*}{ Cofactor } & \multicolumn{2}{|c|}{30 day mortality } & \multicolumn{2}{|c|}{28 day readmission } \\
\hline & Odds ratio $(95 \% \mathrm{Cl})$ & $P$ value & Odds ratio $(95 \% \mathrm{Cl})$ & $P$ value \\
\hline Age (years): & & * & & 0.142 \\
\hline $17-40$ & & & 1.00 & \\
\hline $41-60$ & & & 1.08 (0.90 to 1.28$)$ & 0.426 \\
\hline$>60$ & & & 0.52 (0.24 to 1.11$)$ & 0.091 \\
\hline Operation type: & & 0.049 & & $<0.001$ \\
\hline Gastric bypass & 1.00 & & 1.00 & \\
\hline Gastric banding & 0.22 (0.06 to 0.77$)$ & 0.018 & 0.71 (0.59 to 0.87$)$ & 0.001 \\
\hline Sleeve gastrectomy & $1.60(0.18$ to 13.78$)$ & 0.672 & 1.69 (0.98 to2.93) & 0.061 \\
\hline Female $v$ male sex & & * & 0.81 (0.66 to 1.01$)$ & 0.055 \\
\hline \multicolumn{5}{|l|}{ Comorbidity: } \\
\hline Charlson score $\leq 2$ & 1.00 & & & * \\
\hline Charlson $>2$ & 27.26 (5.53 to 134.49$)$ & $<0.001$ & & \\
\hline Laparoscopy vs open surgery & 0.49 (0.19 to 1.30$)$ & 0.153 & 0.65 (0.54 to 0.78$)$ & 0.001 \\
\hline Provider volume: & & * & & 0.256 \\
\hline Low (1-188 procedures) & & & 1.00 & \\
\hline Medium (189-384 procedures) & & & $0.84(0.68$ to 1.05$)$ & 0.120 \\
\hline High (2384 procedures) & & & $0.88(0.71$ to 1.09$)$ & 0.246 \\
\hline
\end{tabular}

total number of procedures over the study period (2000: gastric bypass 27\% (65/238), gastric banding 73\% (173/238); 2007: gastric bypass 47.7\% (1212/ 2543), gastric banding 49.2\% (1251/2543); $\mathrm{P}<0.001)$. Therefore, we examined gastric bypass and gastric banding separately. Use of the laparoscopic approach was greater among patients who had gastric banding compared with those who had gastric bypass (gastric banding 75.9\% (2768/3649) v gastric bypass 49.5\% (1579/3191); $\mathrm{P}<0.001)$. Furthermore, hospital stay in patients who had a bypass procedure was greater (median length of stay: 5 (3-7) days $(n=3191)$ for gastric bypass $v 2$ (1-3) days $(\mathrm{n}=3649)$ for gastric banding; $\mathrm{P}<0.001)$ and readmission rates postoperatively were higher (28 day readmission: 9.7\% (308/3191) for gastric bypass $v 6.4 \%(232 / 3649)$ for gastric banding; $\mathrm{P}<0.001)$. The risk of postoperative $(30$ day in-hospital) mortality was lower for patients who had laparoscopic banding than for those who had laparoscopic bypass (odds ratio $0.10,95 \%$ confidence interval 0.01 to $0.79 ; \mathrm{P}=0.029$ ).

\section{DISCUSSION}

This population based observational study included 6953 patients who had primary bariatric surgery over an eight year period. This is the first study to analyse national outcomes after surgery for obesity in England. Over the study period, we saw an exponential increase in the volume of bariatric surgery being carried out. By 2007 , largely equivalent volumes of gastric bypass and gastric banding procedures were being done. Women and patients from more socially deprived areas were more likely than men and patients from more affluent areas to have bariatric surgery in NHS hospitals. Patients with higher comorbidities and those from more socially deprived areas showed poorer peri-operative outcome than did other patients. Over the study period, an exponential increase in use of minimal access bariatric surgery occurred. The availability of the laparoscopic approach has been accompanied by a lower readmission rate and shorter duration of inpatient stay.

Over the later part of the study period, the number of bariatric procedures carried out seems to have increased sharply (figure). The reasons for the rapid expansion in provision of bariatric surgery in recent years are manifold. The NICE guideline, advocating the use of surgery for obesity, was published in 2002. ${ }^{11}$ An anticipated delay in changes to everyday practice regularly follows the implementation of such a guideline, given the requirement for training and expansion of services. In addition, agreement must be sought from primary care trusts (the main commissioners in the NHS) that obesity surgery will be funded. In conjunction with the growing level of obesity, as patients become more aware of surgery as a viable treatment option, demand for surgery among morbidly obese patients increases. The expansion of bariatric surgery will begin to tackle the unmet need for bariatric surgery.

As the use of laparoscopy has increased, so have the absolute numbers of bariatric procedures. As with other procedures, a learning curve is associated with bariatric surgery. ${ }^{21}$ In this study, despite the relatively low numbers of procedures done by individual surgeons and providers, we saw no increase in either readmission or mortality rates over the study period, suggesting that laparoscopy has been introduced in a safe manner into the NHS. Indeed, laparoscopy was associated with improved outcome as measured in this study.

\section{Banding versus bypass}

Gastric banding was the most common operation in patients having primary bariatric surgery in England. This study showed a lower readmission rate at 28 days, 
Table 6 | Multiple regression analysis for log transformed length of stay including all patients, with institutional volume and surgeons' volume considered separately*

\begin{tabular}{|c|c|c|c|c|}
\hline \multirow[b]{2}{*}{ Cofactor } & \multicolumn{2}{|c|}{ Trust volume model } & \multicolumn{2}{|c|}{ Surgeon volume model } \\
\hline & Relative risk $(95 \% \mathrm{Cl})$ & $P$ value & Relative risk $(95 \% \mathrm{Cl})$ & $P$ value \\
\hline Female sex (compared with male) & 0.95 (0.92 to 0.99) & 0.007 & 0.95 (0.92 to 0.99) & 0.005 \\
\hline \multicolumn{5}{|l|}{ Operation: } \\
\hline \multicolumn{5}{|l|}{ Gastric bypass (reference)* } \\
\hline Gastric banding & $0.48(0.46$ to 0.49$)$ & $<0.001$ & 0.48 (0.46 to 0.49$)$ & $<0.001$ \\
\hline Sleeve gastrectomy & 1.19 (1.07 to 1.34$)$ & 0.002 & 1.15 (1.03 to 1.29$)$ & 0.012 \\
\hline Laparoscopic $v$ open approach & $0.76(0.62$ to 0.66$)$ & $<0.001$ & 0.63 (0.61 to 0.65$)$ & $<0.001$ \\
\hline \multicolumn{5}{|l|}{ Age cohort (years): } \\
\hline \multicolumn{5}{|l|}{ 17-39 (reference)* } \\
\hline $40-59$ & 1.04 (1.04 to 1.11$)$ & $<0.001$ & 1.07 (1.04 to 1.11$)$ & $<0.001$ \\
\hline$>59$ & 1.02 (1.02 to 1.22$)$ & 0.022 & 1.11 (1.01 to 1.22$)$ & 0.025 \\
\hline \multicolumn{5}{|l|}{ Comorbidity score: } \\
\hline \multicolumn{5}{|l|}{ 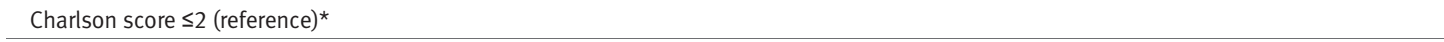 } \\
\hline Charlson score >2 & 1.41 (1.12 to 1.79$)$ & 0.004 & 1.40 (1.11 to 1.77$)$ & 0.004 \\
\hline \multicolumn{5}{|l|}{ Fifth of deprivation (Carstairs index) } \\
\hline \multicolumn{5}{|l|}{1 (least deprived) (reference)* } \\
\hline 2 & 1.05 (1.00 to 1.11$)$ & 0.074 & 1.05 (1.00 to 1.11$)$ & 0.059 \\
\hline 3 & 1.10 (1.04 to 1.16$)$ & 0.001 & 1.10 (1.04 to 1.16$)$ & 0.001 \\
\hline 4 & 1.14 (1.08 to 1.20$)$ & $<0.001$ & 1.13 (1.08 to 1.19$)$ & $<0.001$ \\
\hline 5 (most deprived) & 1.15 (1.10 to 1.21$)$ & $<0.001$ & 1.13 (1.08 to 1.19 ) & $<0.001$ \\
\hline 6 (unclassified) & 0.94 (0.67 to 1.32$)$ & 0.730 & 0.92 (0.66 to 1.29$)$ & 0.634 \\
\hline Discharge year & 0.89 (0.89 to 0.90$)$ & $<0.001$ & $0.90(0.90$ to 0.91$)$ & $<0.001$ \\
\hline \multicolumn{5}{|l|}{ Trust volume/consultant volume: } \\
\hline \multicolumn{5}{|l|}{ Low (reference)* } \\
\hline Medium & 0.87 (0.84 to 0.90$)$ & $<0.001$ & 0.80 (0.77 to 0.83$)$ & $<0.001$ \\
\hline High & 0.77 (0.74 to 0.79$)$ & $<0.001$ & 0.73 (0.70 to 0.76$)$ & $<0.001$ \\
\hline
\end{tabular}

*Length of stay has been logarithmically transformed and exponentiated after analysis.

reduced 30 day and 365 day mortality, and a shorter length of stay after gastric banding compared with gastric bypass and sleeve gastrectomy, although we did not compare postoperative weight loss, morbidity, or revision rates. These findings agree with those of a recent prospective randomised study, in which gastric banding performed more favourably than gastric bypass in terms of mortality at one year and readmission within 30 days. $^{22}$ Lancaster and Hunter found similar results in an observational study using American College of Surgeons National Surgical Quality Improvement Program (ACS-NSQIP) data from the United States. ${ }^{23}$ The NSQIP study also reported a reduced 30 day complication rate among patients having laparoscopic gastric banding compared with laparoscopic gastric bypass.

However, mortality, readmission rates, and length of stay are not the predominant outcome measures of interest to bariatric surgeons. Importantly, gastric bypass has been shown to be superior in terms of percentage postoperative weight loss. ${ }^{24}$ Furthermore, patients selected for gastric bypass may have higher body mass index than those selected for banding. This may explain some of the differences in outcome seen between banding and bypass patients. Clinical registry data will be useful to assess other outcomes from bariatric surgery, such as weight loss and postoperative complications. In the United Kingdom, the
National Bariatric Surgery Registry (NBSR) has recently been launched. This registry will be important in continuing to assess the safety as well as the efficacy of bariatric surgery. In our study, the increase in gastric bypass procedures outstripped that of gastric banding between 2000 and 2008. This trend is diametrically opposed to that in the United States, where gastric banding has increased at a faster rate than bypass. ${ }^{225}$ The reasons for these opposing trends are unclear. The US Food and Drug Administration first approved laparoscopic adjustable gastric bands in 2001. In contrast, England and Europe have a longer history of experience with gastric banding. This historical difference in practice may explain the increasing preference in Europe for bypass, as European surgeons became aware early of the disadvantages of banding. Although banding may be technically less challenging to learn and carry out, the increased weight loss seen after bypass may be an additional driver of the increasing preference in Europe for gastric bypass.

Patient related factors

The predominance of female patients having bariatric surgery seen in this study has been observed in other studies. Psychological factors, such as reduced self esteem and greater rates of depression in female patients, have been reported previously. ${ }^{26}$ Male patients on the other hand may seek bariatric surgery 


\section{WHAT IS ALREADY KNOWN ON THIS TOPIC}

Obesity is an increasing problem

NICE recommends bariatric surgery for morbidly obese patients and overweight patients with coexisting disease who could benefit from weight loss

\section{WHAT THIS STUDY ADDS}

Laparoscopic bariatric surgery has increased exponentially in England, with no increase in mortality or unplanned readmission between 2000 and 2008

Laparoscopic bariatric surgery seems to have been introduced into the English NHS in a safe manner

only when comorbidities become advanced or in the presence of more extreme body mass index. ${ }^{27}$ Although most sleeve gastrectomy patients were also female, we found a greater proportion of male patients $(49 \%)$ in this group than with other types of surgery. Hamoui and colleagues noted a similar proportion of male patients having sleeve gastrectomy. ${ }^{28}$ The preponderance of men in the sleeve gastrectomy group may be explained by this procedure being used more often in patients at higher surgical risk and men possibly presenting with higher body mass index.

Our study shows an inverse relation between socioeconomic status and bariatric surgery (table 1); more patients who had bariatric surgery in NHS hospitals were from areas of lower social deprivation. A higher prevalence of obesity or more extreme body mass index in people from more deprived backgrounds may account for this. The percentage of patients from more affluent backgrounds, however, increased over the study period, which may be a result of the inclusion of patients from the independent sector treated in NHS hospitals or of greater increases in rates of obesity over time in the least deprived groups. No national dataset includes all bariatric patients in the NHS and independent sectors. Private patients treated in the independent sector are not included in the Hospital Episode Statistics dataset. Hence the actual number of more affluent patients having bariatric surgery in England is likely to have been under-represented in this national study.

\section{Variation between institutions}

We found a wide variation in the number of procedures carried out by different institutions over the study period. The definition of high volume provider used in this study differed from that used by the American College of Surgeons. ${ }^{29}$ In our study, we defined a high volume institution as an NHS trust that carried out more than 384 procedures over the study period. The American College of Surgeons defines high volume by centre, with a number of operations exceeding 125 a year needed to qualify as a level $1 \mathrm{a} / 1 \mathrm{~b}$ centre. ${ }^{29} \mathrm{In}$ our study, only $8 / 85(9.4 \%)$ providers met this definition in 2005. Medium volume providers had a lower readmission rate at 28 days and a shorter length of stay compared with low and high volume providers, although the differences in readmission were not statistically significant after regression analyses. However, higher volume centres did more gastric bypass procedures. Complex patients are likely to be referred to tertiary centres for surgery. These tertiary referral centres, with more specialised expertise, are likely to be higher volume centres. Differences in the case mix or complexity of patients not accounted for in this study may explain the variation in readmission rates and length of stay between high and medium volume centres. Use of scoring systems such as the obesity surgery mortality risk score may allow more meaningful comparisons to be drawn between centres operating on patients with different levels of risk. ${ }^{30}$

We considered volume as a categorical variable in this study. This method is similar to that used for other studies looking at volume. ${ }^{1731}$ We defined the thresholds before analysis by using a statistical approach, thereby attempting to negate the bias of selecting thresholds that reach significance. Some researchers have handled volume as a continuous variable..$^{32}$ The output, when a continuous approach is used, is difficult to interpret in a manner that is clinically meaningful.

\section{Study limitations}

The disadvantages of routinely collected datasets have been described elsewhere. ${ }^{143334}$ However, HES data have been used previously to assess the uptake in bariatric surgery in the early years. ${ }^{13}$

HES data are limited in the outcome measures that are available. From this dataset, we could draw no comparisons between procedures in terms of postoperative weight loss, reoperation rate, resolution of comorbidities, or health related quality of life. However, in terms of documenting the national safety of bariatric procedures, mortality, unplanned readmission, and length of stay are important outcome measures.

In this study, we compared gastric bypass, gastric banding, and sleeve gastrectomy. The specific code for sleeve gastrectomy was introduced only in 2006, limiting the numbers of sleeve gastrectomy procedures. We excluded complex bariatric procedures such as biliopancreatic diversion from this study. As such, this study may have underestimated the morbidity associated with bariatric surgery.

\section{Conclusion}

Use of bariatric surgery has increased exponentially in England in recent years. This seems to have occurred safely despite a large increase in uptake of minimal access techniques. Although postoperative weight loss and reoperation rate were not evaluated in this observational population cohort study, patients selected for gastric banding had lower postoperative mortality, readmission rates, and a shorter length of stay than those selected for gastric bypass.

We thank Ahmed R Ahmed, consultant bariatric surgeon, Charing Cross Hospital, Imperial College London and Kamal Nagpal, clinical research fellow, Department of Surgery, Imperial College London for their invaluable advice in this study. Some of the content of the article was presented at the Association of Surgeons of Great Britain and Ireland International Surgical Congress, Liverpool, April 2010. 
Contributors: EMB was involved in the conception and design of the study and drafting of the manuscript and did the analysis of the results. HN was involved in the analysis of the results and drafting of the manuscript. $A B$ was involved in the design of the study, analysis and interpretation of the results, and critically revising the manuscript. AlL was involved in the conception of the study and revising the manuscript. PA and KM were involved in the conception and design of the study and interpreting the results and critically revising the manuscript. OF was involved in the conception and design of the study, analysis and interpretation of the results, and drafting and critically appraising the manuscript. All authors had final approval of the submitted version. OF is the guarantor. Funding: The Dr Foster Unit at Imperial is affiliated with the Centre for Patient Safety and Service Quality at Imperial College Healthcare NHS Trust, which is funded by the National Institute of Health Research. We are grateful for support from the NIHR Biomedical Research Centre funding scheme. The unit is largely funded by a research grant from Dr Foster Intelligence (an independent health service research organisation).

Competing interests: All authors have completed the Unified Competing Interest form at www.icmje.org/coi_disclosure.pdf (available on request from the corresponding author) and declare: no support from any organisation for the submitted work; $\mathrm{AB}$ and PA are part of the Dr Foster Unit, which is largely funded by a research grant from Dr Foster Intelligence (an independent health service research organisation); no other relationships or activities exist that could appear to have influenced the submitted work

Ethical approval: The Patient Information Advisory Group (now the NIGB) granted approval under Section 251 (formerly Section 60). St Mary's local ethics committee has approved the use of these data for research since 2002.

Data sharing: No additional data available.

1 National Task Force on the Prevention and Treatment of Obesity. Overweight, obesity, and health risk. Arch Intern Med 2000;160:898-904.

2 Buchwald H, Oien DM. Metabolic/bariatric surgery worldwide 2008. Obes Surg 2009;19:1605-11.

3 Buchwald H, Buchwald JN. Evolution of operative procedures for the management of morbid obesity 1950-2000. Obes Surg 2002;12:705-17.

4 Le Roux CW, Welbourn R, Werling M, Osborne A, Kokkinos A Laurenius A, et al. Gut hormones as mediators of appetite and weigh loss after Roux-en-Y gastric bypass. Ann Surg 2007;246:780-5.

5 Schirmer B. Laparoscopic bariatric surgery. Surg Endosc 2006;20(suppl 2):450-5S.

6 Must A, Spadano J, Coakley EH, Field AE, Colditz G, Dietz WH. The disease burden associated with overweight and obesity. JAMA 1999;282:1523-9.

7 Buchwald H, Avidor Y, Braunwald E, Jensen MD, Pories W, Fahrbach K, et al. Bariatric surgery: a systematic review and metaanalysis. JAMA 2004:292:1724-37.

8 Christou NV, Sampalis IS, Liberman M, Look D, Auger S, McLean AP, et al. Surgery decreases long-term mortality, morbidity, and health care use in morbidly obese patients. Ann Surg 2004;240:416-23.

9 Adams TD, Gress RE, Smith SC, Halverson RC, Simper SC, Rosamond WD, et al. Long-term mortality after gastric bypass surgery. N Engl / Med 2007;357:753-61.

10 Sjostrom L, Narbro K, Sjostrom CD, Karason K, Larsson B, Wedel H, et al. Effects of bariatric surgery on mortality in Swedish obese subjects. N Engl J Med 2007;357:741-52.

11 National Institute for Health and Clinical Excellence. Guide on the use of surgery to aid weight reduction for people with morbid obesity. NICE, 2002.

12 Colquitt J, Clegg A, Loveman E, Royle P, Sidhu MK. Surgery for morbid obesity. Cochrane Database Syst Rev 2005;4:CD003641.

13 Ells LJ, Macknight N, Wilkinson JR. Obesity surgery in England: an examination of the health episode statistics 1996-2005. Obes Surg 2007;17:400-5.

14 Faiz O, Warusavitarne J, Bottle A, Tekkis PP, Darzi AW, Kennedy RH. Laparoscopically assisted vs open elective colonic and rectal resection: a comparison of outcomes in English National Health Service trusts between 1996 and 2006. Dis Colon Rectum 2009;52:1695-704.
15 Gill LE, Goldacre MJ. English national record linkage of Hospita Episode Statistics and death registration records: Report to the Department of Health, 2003. National Centre for Health Outcomes Development, Unit of Health-care Epidemiology, University of Oxford, 2003 (available at http://nchod.uhce.ox.ac.uk/NCHOD\%200xford $\% 20$ E5\%20Report\%201st\%20Feb_VerAM2.pdf).

16 Charlson ME, Pompei P, Ales KL, MacKenzie CR. A new method of classifying prognostic comorbidity in longitudinal studies: development and validation. J Chronic Dis 1987;40:373-83.

17 Borowski DW, Kelly SB, Bradburn DM, Wilson RG, Gunn A, Ratcliffe AA. Impact of surgeon volume and specialization on shortterm outcomes in colorectal cancer surgery. Br J Surg 2007;94:880-9.

18 Schrag D, Cramer LD, Bach PB, Cohen AM, Warren JL, Begg CB. Influence of hospital procedure volume on outcomes following surgery for colon cancer. JAMA 2000;284:3028-35.

19 Schrag D, Panageas KS, Riedel E, Cramer LD, Guillem JG, Bach PB, et al. Hospital and surgeon procedure volume as predictors of outcome following rectal cancer resection. Ann Surg 2002;236:583-92

20 Kennedy ED, Rothwell DM, Cohen Z, McLeod RS. Increased experience and surgical technique lead to improved outcome after ileal pouch-anal anastomosis: a population-based study. Dis Colon Rectum 2006:49:958-65.

21 Schauer P, Ikramuddin S, Hamad G, Gourash W. The learning curve for laparoscopic Roux-en-Y gastric bypass is 100 cases. Surg Endosc 2003;17:212-5.

22 Nguyen NT, Slone JA, Nguyen XM, Hartman JS, Hoyt DB. A prospective randomized trial of laparoscopic gastric bypass versus laparoscopic adjustable gastric banding for the treatment of morbid obesity: outcomes, quality of life, and costs. Ann Surg 2009; published online 27 August.

23 Lancaster RT, Hutter MM. Bands and bypasses: 30-day morbidity and mortality of bariatric surgical procedures as assessed by prospective, multi-center, risk-adjusted ACS-NSQIP data. Surg Endosc 2008;22:2554-63.

24 Garb J, Welch G, Zagarins S, Kuhn J, Romanelli J. Bariatric surgery for the treatment of morbid obesity: a meta-analysis of weight loss outcomes for laparoscopic adjustable gastric banding and laparoscopic gastric bypass. Obes Surg 2009;19:1447-55

25 Hinojosa MW, Varela JE, Parikh D, Smith BR, Nguyen XM, Nguyen NT. National trends in use and outcome of laparoscopic adjustable gastric banding. Surg Obes Relat Dis 2009;5:150-5

26 Kolotkin RL, Crosby RD, Gress RE, Hunt SC, Engel SG, Adams TD. Health and health-related quality of life: differences between men and women who seek gastric bypass surgery. Surg Obes Relat Dis 2008;4:651-8.

27 Rand CS, Kuldau JM. Morbid obesity: a comparison between a general population and obesity surgery patients. Int J Obes Relat Metab Disord 1993;17:657-61.

28 Hamoui N, Anthone GJ, Kaufman HS, Crookes PF. Sleeve gastrectomy in the high-risk patient. Obes Surg 2006;16:1445-9.

29 Schirmer B, Jones DB. The American College of Surgeons Bariatric Surgery Center Network: establishing standards. Bull Am Coll Surg 2007;92:21-7.

30 DeMaria El, Murr M, Byrne TK, Blackstone R, Grant IP, Budak A, et al. Validation of the obesity surgery mortality risk score in a multicente study proves it stratifies mortality risk in patients undergoing gastric bypass for morbid obesity. Ann Surg 2007;246:578-82.

31 Faiz O, Brown T, Bottle A, Burns EM, Darzi AW, Aylin P. Impact of hospital institutional volume on postoperative mortality after major emergency colorectal surgery in English National Health Service trusts, 2001 to 2005. Dis Colon Rectum 2010;53:393-401.

32 Spiegelhalter DJ. Mortality and volume of cases in paediatric cardiac surgery: retrospective study based on routinely collected data. BMJ 2002;324:261-3.

33 Aylin P, Lees T, Baker S, Prytherch D, Ashley S. Descriptive study comparing routine hospital administrative data with the Vascular Society of Great Britain and Ireland's national vascular database. Eur J Vasc Endovasc Surg 2007;33:461-5.

34 Aylin P, Bottle A, Elliott P, Jarman B. Surgical mortality: hospital episode statistics v central cardiac audit database. $B M J$ 2007;335:839.

Accepted: 11 June 2010 